\title{
Financial Benefit of Using Crop Protection Decision Rules Over Systematic Spraying Strategies
}

\author{
F. Fabre, M. Plantegenest, and J. Yuen
}

First author: INRA, UR 407, Unité de Pathologie Végétale, BP 94, 84143 Avignon Cedex, France; second author: INRA/Agrocampus Rennes, UMR 1099, Unité Mixte de Recherche BiO3P, 65 rue de Saint-Brieuc, CS 84215, 35042 Rennes, France; and third author: Department of Forest Mycology and Pathology, Swedish University of Agricultural Sciences, Box 7026 , SE 75007 Uppsala, Sweden.

Accepted for publication 15 June 2007.

\begin{abstract}
Fabre, F., Plantegenest, M., and Yuen, J. 2007. Financial benefit of using crop protection decision rules over systematic spraying strategies. Phytopathology 97:1484-1490.

Decision rule models are considered to be one of the main cornerstones of the implementation of integrated pest management (IPM) programs. Even if the need for such programs to offer cost advantages over conventional strategies is a major incentive for IPM adoption, few studies focus on this financial dimension. In this article, a modeling approach of the response of a pathosystem to a disease control method and of the predictive performance of decision rules is used to explore how some

cost of systematic strategies in several different scenarios, the models developed here showed strong effects of different pathosystems and decision rules on financial benefits. The number of production situations where decision rules are of interest is highly correlated with their accuracy. However, because of the inescapable trade-offs between decision rule accuracy and limiting factors such as its user-friendly characteristics, the use of decision rules is unlikely to reduce costs to $<70 \%$ of the costs of systemic strategies. In more general terms, this study provides quantitative guidelines on the financial advantage that decision rules can offer in plant protection as well as a better understanding of their potential usefulness.
\end{abstract} basic factors act on the likelihood of adoption of decision rule models strategies (such as using an IPM system) over systematic strategies (such as systematic-spraying and never-spraying strategies). Even if the average cost of using the decision rule strategies is always lower than the average
Additional keywords: control strategies, receiver operating characteristic (ROC) curve.
When making a decision on whether or not to apply a pesticide, a farmer has to choose between three possible control strategies. These are to (i) spray prophylactically, (ii) never spray, or (iii) spray according to the recommendations of tactical models $(1,27)$. Tactical models are designed to advise farmers of the need and, sometimes, timing of applying crop protection measures (37). The simplest tactical models are decision rules designed to provide farmers with binary advice: "Yes, treatment is needed" or "No, it's not worth the trouble." Such decision rules associate a risk indicator and a decision threshold. A risk indicator is a measurement or a calculation performed to assess the risk that a pest or a disease will produce severe yield losses in a crop. Indicators range from simple sampling models (10) that estimate disease intensity in a field to complex forecast models that take into account the influence of the environment on the pathogen and predict the likelihood of future disease development $(16,20)$. Complex indicators may be composed of several rules or calculations that have been referred to as risk algorithms (39). Whatever these levels of complexity, the necessity to apply a treatment is evaluated by comparing the value of the indicator assessed in a given field $(I)$ to a decision threshold $(I s)$, and a treatment is recommended if the indicator value exceeds the decision threshold $(I>I s)$. For a given indicator $I$, various decision rules can be defined according to the value of $I s$ selected. However, a single rule usually will best fit the management goals of the decision maker (34).

Corresponding author: F. Fabre; E-mail address: frederic.fabre@avignon.inra.fr

doi:10.1094/PHYTO-97-11-1484

(c) 2007 The American Phytopathological Society
Tactical models are considered to be one of the main cornerstones of the implementation of integrated pest management (IPM) programs. Accordingly, many research projects are aimed at development of such systems. However, until now, there has been a low level of adoption of tactical models by farmers (15, 22,37). Incentives for IPM adoption are very diverse. Although environmental issues were a driving force behind the development of IPM, they apparently act as a minor incentive with regard to pest control decisions by farmers (14). On the other hand, the need for IPM programs to offer cost advantages over conventional strategies is often cited as a major incentive for IPM adoption (38). Accordingly, one can suppose that the likelihood of adoption of tactical models by farmers is highly correlated with the financial advantage that this strategy can offer over conventional crop protection strategies. Although the financial dimension of disease management probably is quite important to those who decide whether to use IPM, it often is not considered by those that develop the models (13).

The profitability of any crop protection strategy depends on (i) the prevalence of the pest or disease considered; (ii) the potential yield losses associated to this pest or disease; (iii) the cost and efficiency of the protection measure; and (iv), in the case of tactical models, on their accuracy (i.e., their predictive performance). In this article, using a modeling approach based on costbenefit and receiver operating characteristic (ROC) curve theories, we will explore how these four factors act on the likelihood of adoption of decision rule models, one important category of tactical models. The focus, in particular, is to estimate what financial advantage can be reached given the level of accuracy that can be achieved given the type of data that is usually available combined with user-friendly decision rules. 


\section{THEORY AND APPROCHES}

The first part of this section is devoted to modeling the average cost of systematic spraying strategies and the average cost of spraying strategies based on decision rules as a function of some basic pathosystem characteristics and decision rule accuracy. Then, methods for estimating the financial benefit of decision rules are derived.

Modeling the average cost of systematic strategies. Characterization of the financial response of a pathosystem to a treatment. Let Ctrt be the average cost of the treatment against a particular pest or disease. Let $T$ be a binary random variable: $T=T+$ for the fields, termed hereafter cases, where the cost of the yield losses due to the considered pest or disease is greater (or equal) to Ctrt (i.e., fields where a treatment is justified in purely cost terms) and $T=T$ - for the fields, termed hereafter controls, where the costs of the yield losses due to the considered pest or disease are lower than Ctrt (i.e., fields where a treatment is not justified). The average cost of the yield losses due to the considered pest or disease for the cases and the controls are Cmax and Cmin, respectively. It is assumed that $0<C \min <C \max$. Moreover, the proportion of cases in the sample of fields, termed prevalence (Prev), is the a priori probability that a treatment is required.

Finally, we define two new variables: (i) $\alpha$, the extent of the potential yield loss defined such that $\alpha=C \max / C \min$, and (ii) $\beta$, the relative cost of the protection measure defined as the ratio between (Ctrt - Cmin) and (Cmax - Cmin). Thus, $C$ trt $=C \min \times[1+\beta \times(\alpha-1)]$, with $0<\beta<1$ (Fig. 1). Large values of $\alpha$ characterize pathosystems with high potential yield loss due to the pest or disease of interest. Large values of $\beta$ characterize control measures expensive compared with yield loss difference between the cases and the controls. From the previous considerations, in this study, the financial response of a pathosystem to a treatment is modeled with four variables: Prev, Cmin, $\alpha$, and $\beta$.

Average cost of systematic strategies. The average cost resulting from the use of the systematic-spraying strategy is $C_{S S}=C t r t$. From the previous notation, it can be derived that

$$
C_{S S}(C \min , \alpha, \beta)=C \min \times[1+\beta \times(\alpha-1)]
$$

Similarly, the average cost resulting from the use of the neverspray strategy is $C_{N S}=$ Prev $\times$ Cmax $+(1-$ Prev $) \times C m i n$. From the previous notation, we derive

$$
C_{N S}(\text { Cmin }, \alpha, \operatorname{Prev})=C \min \times[\operatorname{Prev} \times(\alpha-1)+1]
$$

By solving the inequality $C_{S S}(C \min , \alpha, \beta)>C_{N S}(C \min , \alpha$, Prev), we can see that the best systematic strategy is the systematic-spraying strategy if Prev $>\beta$ and the never-spray strategy if not.

Modeling the average cost of decision rule strategy. Characterization of the accuracy of decision rules. The financial interest of spraying according to a decision rule depends on its degree of accuracy. The accuracy of decision rules used to distinguish between binary events (e.g., $\mathrm{T}+$ or $\mathrm{T}-$ ) can be assessed independently of the prevalence of the cases (Prev) in terms of their sensitivity $(S e)$ and specificity $(S p)(11,12,24,28,40)$. Se, defined as $p(I>I s \mid T+)$, is the probability of true positive decisions. Similarly, $S p$, defined as $S p=p(I \leq I S \mid T-)$, corresponds to the probability of true negative decisions. For most indicators, $S e$ and $S p$ are linked by a function $f$ such that $S e=f(1-S p)$, where $f$ is monotonous, convex, and always joins the points 0,0 and 1,1 . This function, named the ROC curve, is a plot of $S e$ against (1 $S p$ ) for all the possible values of the decision threshold $I s$ $(24,25,28)$. ROC curve analysis has become quite common in crop protection $(1,12,21,36,40)$.

When the distribution of the value of an indicator $(I)$ in the cases and the controls are Gaussian (or can be transformed to Gaussian ones by a monotonic transformation) (25), the model of
Lloyd (19) can be used to describe the ROC curve of an indicator $(I)$ :

$$
S e=\left[1+e^{-\Delta} \times\left[(1-S p)^{-\mu}-1\right]\right]^{-\frac{1}{\mu}}
$$

where $\mu$ is a parameter describing the asymmetry of the curve on the minor axis and $\Delta$ a shift parameter.

In Lloyd's model, the parameter $\Delta$ is closely related to the area under the ROC curve (AUC) of the indicator (Fig. 2). The AUC is a standardized measure of the overall accuracy of indicators: the closer to 1 the AUC of an indicator is, the better this indicator is $(12,32,33)$. An indicator characterized by a ROC curve that comes near to the 0,1 point (where sensitivity and specificity are both equal to 1) is a near-perfect indicator. The opposite of this is an ROC curve of an indicator that is unable to discriminate fields where a treatment is needed from those where it is unnecessary. This would be a straight line joining the 0,0 point to the 1,1 point (the "no discrimination" line), and the AUC would be 0.5 .

Average cost of the optimal decision rule strategy. The average cost resulting from the use of a decision rule strategy $\left(C_{D R}\right)$ of given sensitivity $(\mathrm{Se})$ and specificity $(\mathrm{Sp})$ is

$$
\begin{gathered}
C_{D R}=\text { Prev } \times[C t r t \times S e+C \max \times(1-S e)]+ \\
(1-\text { Prev }) \times[C \min \times S p+C t r t \times(1-S p)]
\end{gathered}
$$

Basically, $C_{D R}$ is calculated from a sum of the average costs (Cmin, Cmax, and Ctrt) weighted by the probability of the four possible combinations of the true need to spray and the decision to spray or not spray. The true need to spray is based on the value of $T(T+$ or $T-)$, and the decision to spray is a based on Prev, Se, and $S p$ (39). From this weighted sum, and estimating the ROC curve using the model of Lloyd, we can obtain

$$
\begin{aligned}
& C_{D R}(\text { Cmin }, \alpha, \beta, \text { Prev }, \Delta, \mu, S p)=\text { Cmin } \times \text { Prev } \times \\
& {[f(\Delta, \mu, S p) \times[1+\beta \times(\alpha-1)-\alpha]+\alpha]+\operatorname{Cmin} \times(1-\text { Prev }) \times} \\
& {[S p+[1+\beta \times(\alpha-1)] \times(1-S p)]}
\end{aligned}
$$

Thus, we can describe the cost of using a decision rule given a pathosystem (i.e., a set of values for Prev, Cmin, $\alpha$ and $\beta$ ) and an indicator (i.e., a set of values for $\Delta$ and $\mu$ ). Several different decision rules can be chosen based on the value of the decision threshold $I s$, but a single one, the optimal decision rule, will minimize $C_{D R}$. This optimal decision rule is such that

$$
\frac{d C_{D R}(S p)}{d S p}=0 \Leftrightarrow \frac{d S e}{d(1-S p)}=\frac{1-\text { Prev }}{\text { Prev }} \times \frac{\beta}{1-\beta}
$$

where $d S e / d(1-S p)$ is the slope of the ROC curve of the indicator $I(13,24)$. The average cost resulting from the use of the optimal decision rule strategy will be denoted $C_{D R o p t}$.

Average cost of perfect strategy. A perfect strategy always recommends a treatment when necessary and never when it is not needed (both sensitivity and specificity are equal to 1 ). Its average

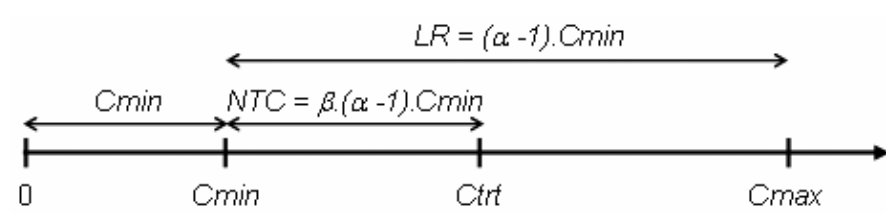

Fig. 1. Cost line representing the financial response of a pathosystem to a treatment in absolute and relative amounts and showing derivation of $\alpha$ and $\beta$. The average cost of the yield losses due to the considered pest or disease for fields where a treatment is not required is Cmin and for fields where a treatment is necessary is Cmax. Parameter $\alpha$ is a measure of the extent of the potential yield loss caused by the pest or disease, where $\alpha=C \mathrm{~min} / \mathrm{Cmax}$ with Cmin $>0$. The difference between Cmax and Cmin is the loss range (LR). Ctrt is the average cost of the treatment. The net treatment costs $(N T C)$ is the difference between Ctrt and Cmin, and $\beta$ is the ratio of NTC and $L R(0<\beta<1)$. 
costs is $C_{P}=$ Prev $\times$ Ctrt $+(1-$ Prev $) \times$ Cmin. Using our previous notation, it can be written as

$$
C_{P}(\text { Cmin }, \alpha, \beta, \text { Prev })=\operatorname{Cmin} \times[1+\operatorname{Prev} \times \beta \times(\alpha-1)]
$$

This strategy was considered mainly for research purposes in order to study the potential interest of improving indicators.

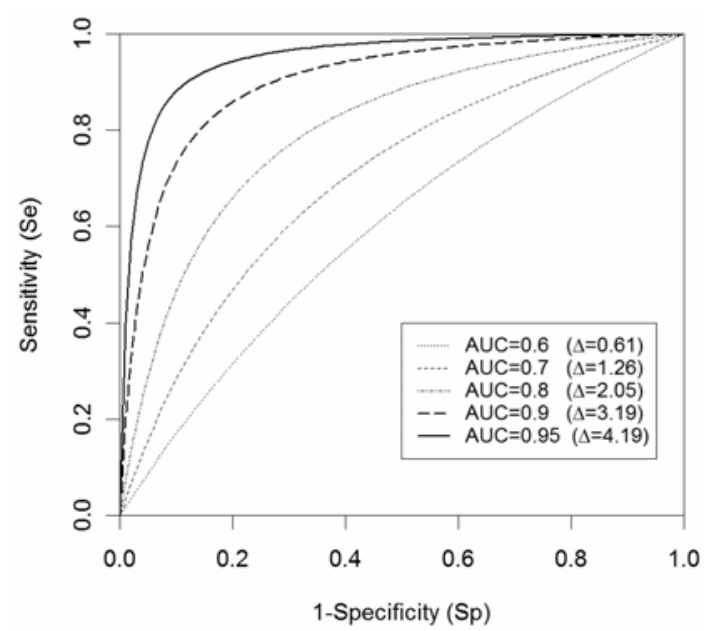

Fig. 2. Receiver operating characteristic (ROC) curves fitted with the model of Lloyd for five values of the shift parameter $\Delta$ corresponding to five values of the area under the ROC curve (AUC): AUC $=0.6(\Delta=0.61)$, AUC $=0.7(\Delta=$ $1.26), \mathrm{AUC}=0.8(\Delta=2.05), \mathrm{AUC}=0.9(\Delta=3.19)$. and $\mathrm{AUC}=0.95(\Delta=$ 4.19). The asymmetry parameter $(\mu)$ is set to 1 .
Financial advantage provided by the decision rule strategy. Cost reduction. The financial advantage of using a decision rule strategy is assessed as the cost reduction $(C R)$, which is the ratio of the costs from using an optimal decision rule compared with the costs of using the better systematic strategy. $C R$ is defined as follows:

$$
\begin{aligned}
& C R(\alpha, \beta, \text { Prev }, \Delta, \mu)=\frac{C_{D R o p t}(\operatorname{Cmin}, \alpha \beta, \text { Prev }, \Delta, \mu)}{C_{S S}(\text { Cmin }, \alpha, \beta)} \text { if Prev }>\beta \\
& C R(\alpha, \beta, \text { Prev }, \Delta, \mu)=\frac{C_{D R o p t}(\text { Cmin }, \alpha \beta, \text { Prev }, \Delta, \mu)}{C_{N S}(\text { Cmin }, \alpha, \text { Prev })} \text { if Prev } \leq \beta
\end{aligned}
$$

Notice that, as Cmin can be factorized in each numerator and denominator, $C R$ doesn't depend on Cmin.

Overall cost reduction. We defined the overall cost reduction $(O C R)$ as

$$
\operatorname{OCR}(\alpha, \beta, \Delta, \mu)=1-\int_{0}^{1} C R(\alpha, \beta, \operatorname{Prev}, \Delta, \mu) \times d \operatorname{Prev}
$$

$O C R$ is a measure of the financial interest of using a $D R$ strategy for all possible value of Prev. On a graph representing $C R$ as a function of Prev (Fig. 3), OCR is the area above this curve and the line $C R=1$.

Numerical simulations. A set of functions were developed using the statistical package $\mathrm{R}$ to numerically explore the effects of the five parameters Prev, $\alpha, \beta, \mu$, and $\Delta$ on the value of $C R$ and $O C R$. Based on bibliographic data, ranges of variation reflecting a wide diversity of pathosystem or decision rules were used to
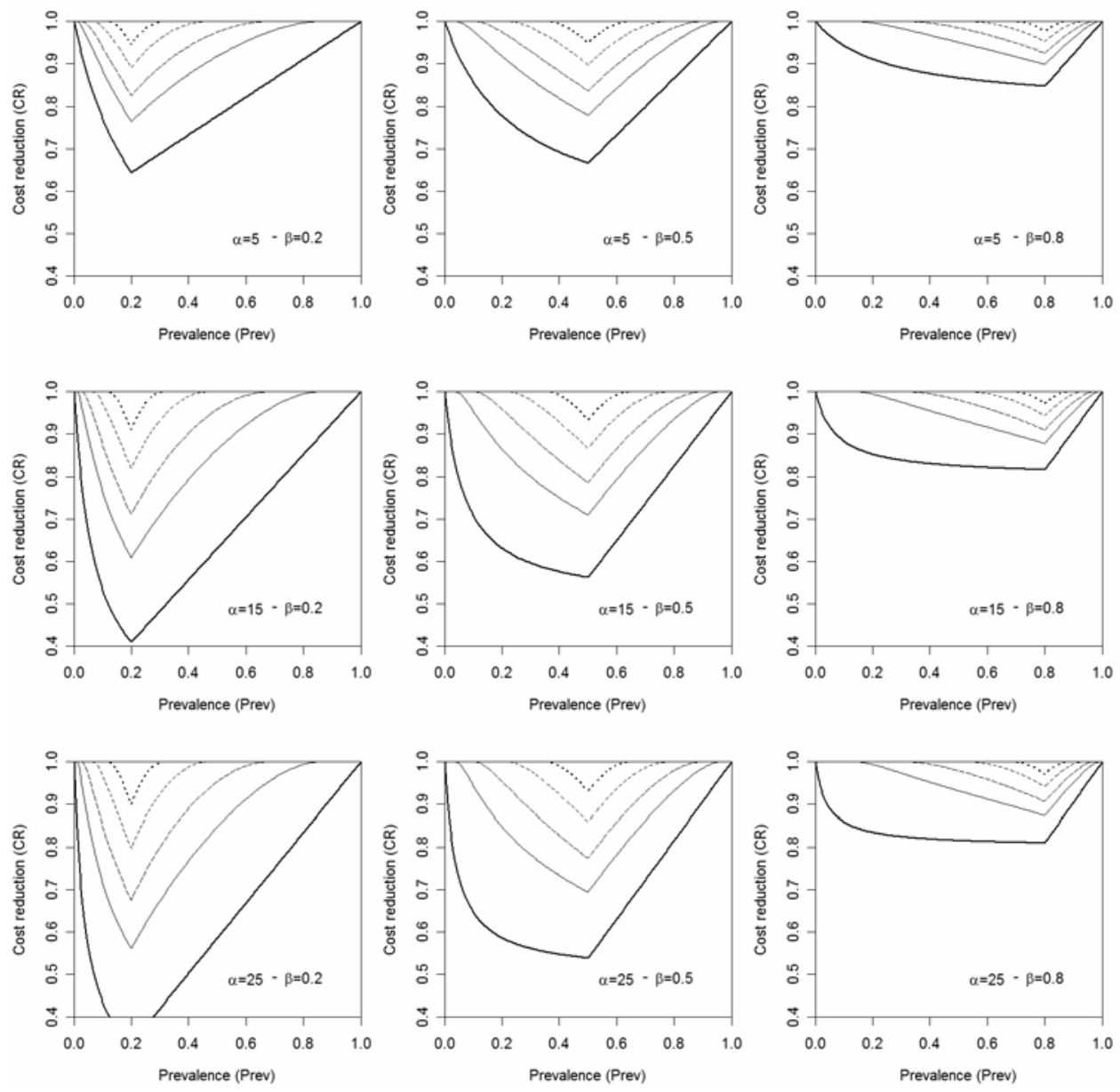

Fig. 3. Cost reduction $(C R)$ resulting from the use of a decision rule strategy over the better systematic strategy as a function of disease prevalence (Prev) for five indicators of increasing overall accuracy (AUC $=-\cdots 0.6,--0.7,----0.8,-0.9$, and $-1-$ ) Values of $C R$ are illustrated for three levels of potential yield loss $(\alpha=5,15$, and 25$)$ and of the relative cost of the treatment $(\beta=0.2,0.5$, and 0.8$)$. 
assign to each factor. $C R$ was assessed for all possible values of the parameters in their ranges with a $5.10^{-3}$ step. For AUC $=1$, calculations were done by substituting $C_{D R o p t}$ with $C_{P}$ in the expression of $C R$.

\section{RESULTS}

Assignment of range of variation to the parameters studied. In our modeling framework, the $C R$ provided by a decision rule strategy over the best systematic strategy depends on five parameters. Three parameters characterize a pathosystem: (i) $\alpha$, the extent of the potential yield loss; (ii) $\beta$, the relative cost of the treatment, and (iii) Prev, the prevalence of the major yield loss situations. Some studies $(1,6,17,18,21)$ reporting long-term data sets from which these parameters can be estimated were used to assign a range of variation to $\alpha$ between 1 and 25. This range encompasses several pathosystems and production situations (Table 1). For $\beta$ and Prev, ranges of variation between 0 and 1 encompassing all possible values of relative treatment cost were defined.

In the model of Lloyd, two parameters characterize the overall accuracy (AUC) of an indicator: (i) $\mu$, an asymmetry parameter and (ii) $\Delta$, a shift parameter. A preliminary analysis indicates that $C R$ is only slightly sensitive to $\mu$ (not shown). This parameter then was set to 1 (i.e., only indicators with ROC curve symmetrical on the minor axis were considered-this arises when the variance of the distribution of the indicator values of the cases and the controls are equal). The range of variation of $\Delta$ was defined in order to obtain AUC ranging from 0.55 to $1(0.301 \leq \Delta \leq 6.277)$. This range encompasses the AUC of most crop protection decision rules. Bibliographic data on several pathosystems (Barley yellow dwarf virus on barley [5], fire blight on apple and pear [3], Sclerotinia stem rot on oilseed rape [21,40], and apple scab on apple [36]) indicate that AUC values of crop protection decision rules range from 0.6 to 0.9 and rarely exceed this value. This is mainly because modelers have to face many uncertainties when modeling a biological system (e.g., processes involved and availability of necessary microclimatic data) and also often try to develop user-friendly models.

The average cost of the optimal decision rule strategy was always lower (or at least equal) to those of systematic strategies (either the systematic spraying strategy or the never spray strategy). However $C R$ provided by optimal decision rule strategies were strongly affected by variation in pathosystem and decision rules parameters.

Effect of disease prevalence (Prev). As expected, $C R$ depended strongly on the prevalence of major yield loss (Prev). When a pest was rare (i.e., $(1-$ Prev $) /$ Prev is large), the optimal decision threshold $I s$ is shifted to the lower left part of the ROC curve where the slope is large. The selected decision rule will consequently have a high specificity $(S p)$ and a low sensitivity (Se) (Fig. 2). Only decision rules with a very good level of accuracy (both high $S e$ and $S p$ ) significantly reduce the average cost of decision rule strategy $\left(C_{D R}\right)$ compared with the average cost of the never-spray strategy $\left(C_{N S}\right)$. The same phenomena arose for the average cost of decision rules strategy developed for very common pests or diseases when compared with the average cost of a systematic-spraying strategy.

Effect of the extent of the potential yield loss $(\alpha)$. For given relative cost of treatment $(\beta)$ and overall indicator accuracy (AUC), both $C R$ (Fig. 3) and $O C R$ (Fig. 4) increased with the extent of the potential yield loss $(\alpha)$. This relationship was not linear: $O C R$ was much more sensitive to $\alpha$ for values $<10$ compared with higher ones (Fig. 4B).

Effect of the relative cost of a treatment $(\beta)$. The relative cost of a treatment $(\beta)$ determines the value of disease prevalence (Prev) maximizing $C R$ (Fig. 3). The $O C R$ strongly depended on $\beta$. Values of $\beta$ ranging from 0.1 to 0.6 lead to higher $O C R$ s (Fig. 5). Outside of this range, only highly accurate indicators provided
A
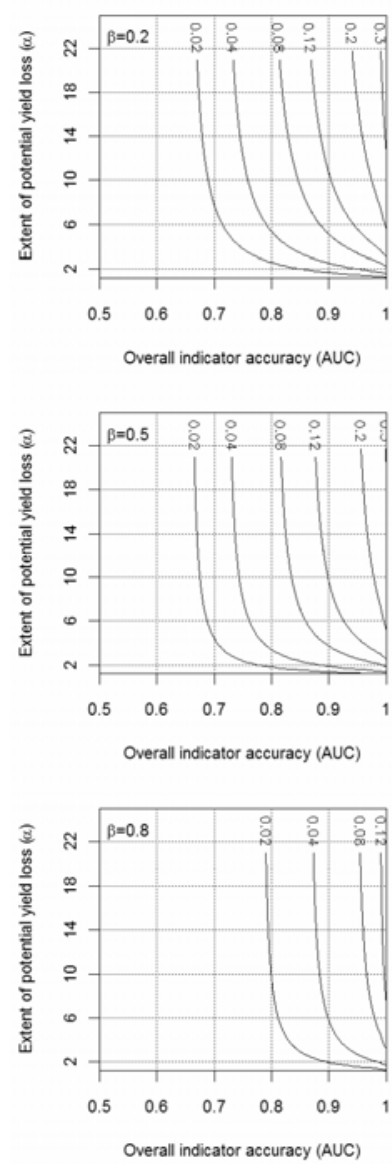

B
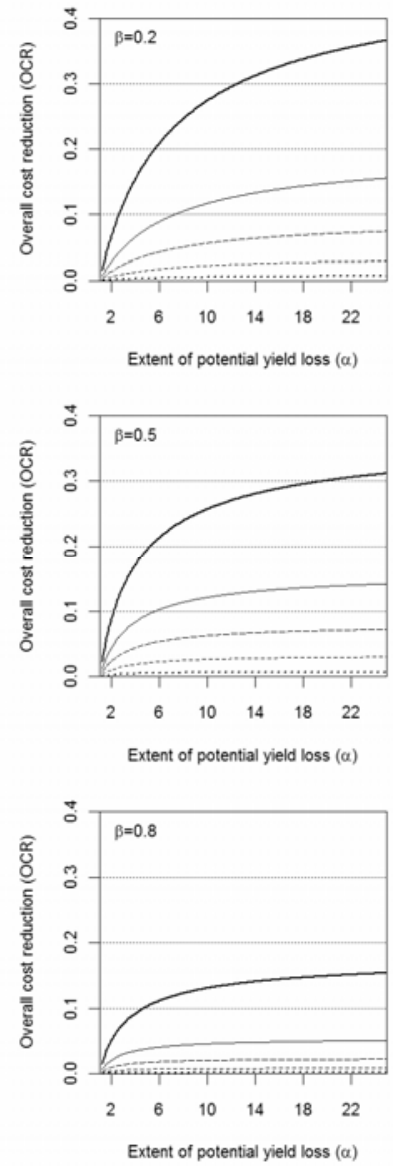

Fig. 4. Effects of the overall indicators accuracy (area under the receiver operating characteristic curve $[\mathrm{AUC}]$ ) and the extent of the potential yield loss caused by the considered pest or disease $(\alpha)$ on the overall cost reduction (OCR) for three values of $\beta(0.2,0.5$, and 0.8$)$. A, OCR as a function of AUC ( $x$ axis) and $\alpha$ ( $y$ axis). B, OCR as a function of $\alpha$ for five levels of AUC. AUC $=-\cdots 0.6,-\cdots 0.7,---0.8,-0.9$, and -1 .

TABLE 1. Range of variation of $C t r t, C \min , C \max , \alpha$, and $\beta$ in some pathosystems ${ }^{\mathrm{a}}$

\begin{tabular}{|c|c|c|c|c|c|c|}
\hline \multirow[b]{2}{*}{ Crop, pest } & \multicolumn{3}{|c|}{ Costs (kg/ha) } & \multirow[b]{2}{*}{$\alpha$} & \multirow[b]{2}{*}{$\beta$} & \multirow[b]{2}{*}{ Reference } \\
\hline & Ctrt & Cmin & Cmax & & & \\
\hline Oilseed rape, Sclerotinia stem rot & 155 (triazole) & 21 & 421 & 20 & 0.33 & 21 \\
\hline Barley, Barley yellow dwarf virus & 500 (pyrethroids) & 122 & 2,120 & 17 & 0.19 & 6 \\
\hline Oat, frit fly & 200 (pyrethroids) & 50 & 700 & 14 & 0.23 & 18 \\
\hline Wheat, grain aphid & 300 (pyrethroids) & 153 & 574 & 3.8 & 0.35 & 17 \\
\hline
\end{tabular}

${ }^{a}$ All costs $(C t r t=$ average treatment cost, $C$ min $=$ average cost of yield losses in the controls, and Cmax $=$ average cost of yield losses in the cases) are expressed in $\mathrm{kg} / \mathrm{ha}$ of yield losses. The extent of the potential yield loss $(\alpha)$ is defined as Cmax/Cmin and the relative cost of the treatment $(\beta)$ is defined as $(C t r t-$ Cmin $) /(\operatorname{Cmax}-\operatorname{Cmin})$. 
substantial financial interest when compared with the best systematic strategy. Accordingly, for a given pathosystem, a single value of $\beta$ maximized the financial interest of using a decision rule strategy (Fig. 5B).

Effect of overall indicator accuracy (AUC). The nine plots of Figure 3 explored a large diversity of pathosystems. It is interesting to note that, for all the AUC in the range of accuracy of most crop protection decision rules (range of 0.6 to 0.9 ) and all pathosystems illustrated, $C R$ is rarely $<0.7$, which corresponds to an average cost saving of $30 \%$. Figure 3 also clearly illustrates that, when Prev departs from the value of $\beta$ that minimized $C R, C R$ increased more or less rapidly according to the overall indicator accuracy (AUC). When comparing $C R$ obtained with indicators in the AUC range (0.6 to 0.9$)$ with $C R$ obtained with a perfect indicator (Fig. 3) $(\mathrm{AUC}=1)$, it appears that $C R$ does not depend linearly on AUC: improvements in $C R$ are increasingly more important when AUC tends toward 1.

\section{DISCUSSION}

Effect of disease prevalence (Prev). In the plant protection context, Prev reflects the production situation of a field (i.e., the set of physical, biological, and socioeconomic factors that determine agricultural production) $(4,26,30)$. As was expected, $C R$ was very sensitive to Prev. For rare pests or common pests, the optimum decision threshold is shifted to the ends of the ROC curve. Thus, decision rules with very good accuracy (in terms of
A
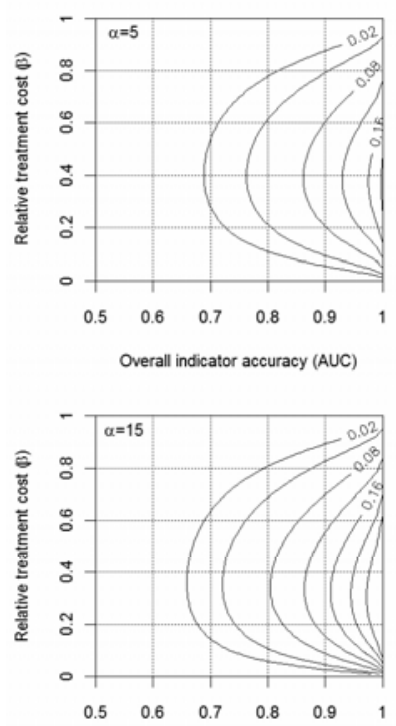

Overall indicator accuracy (AUC)

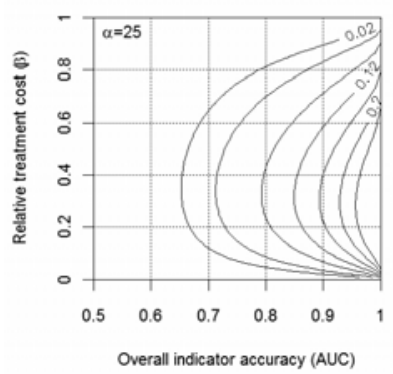

B
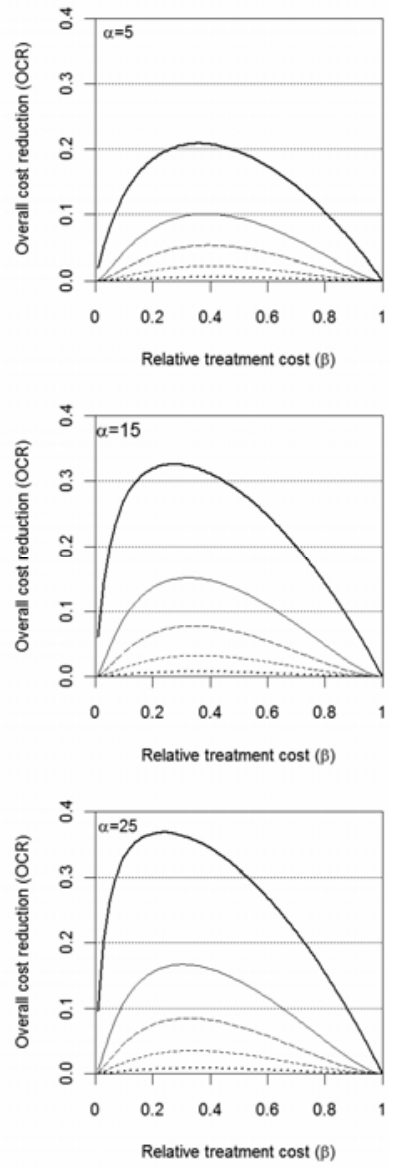

Fig. 5. Effects of the overall indicators accuracy (area under the receiver operating characteristic curve $[\mathrm{AUC}])$ and the relative cost of the treatment $(\beta)$ on the overall cost reduction (OCR) for three values of $\alpha(5,15$, and 25). A, OCR as a function of AUC ( $x$ axis) and $\beta$ ( $y$ axis). B, OCR as a function of $\beta$ for five levels of AUC. AUC = - - 0.6, - - 0.7, - - $-0.8,-0.9$, and -1 . both specificity and sensitivity) are required to offer any benefits over the no-treatment strategy (for rare pests) or the always-treat strategy (for common pests). The financial interest of using a decision rule strategy is high only for intermediate levels of prevalence of the pest or disease. $C R$ is minimal (and so the financial motivation for using the decision rule strategy is maximal) when Prev $=\beta$ (Fig. 3). These conclusions are in agreement with those of Murthaugh (28) and Yuen and Hughes (39), who analyzed the role of Prev on the usefulness of rule-based systems from the point of view of their predictive values.

Effect of the extent of the potential yield loss $(\alpha)$. The nonlinear relationship between $O C R$ and $\alpha$ implies a sensitivity to the extent of the potential yield loss caused by the pathosystem. Although it might be obvious that a $D R$ strategy would be more interesting for a moderately destructive pathogen compared with a less destructive one, it is not clear that the gain from going from a moderately destructive pathogen to a more destructive one would be so modest.

Effect of the relative cost of a treatment $(\beta)$. In our model, $\beta$ is a measure of Ctrt. Ctrt usually includes direct costs such as pesticide, labor, and tractor wheel costs. However, one also could be interested in including indirect costs such as the environmental cost of the treatment $(9,29)$. The dependency of $O C R$ on $\beta$ and the existence of a value of $\beta$ that would optimize the financial interest in using decision rules makes this a possible way to affect the adoption of tactical models. This is because $\beta$, unlike Prev and $\alpha$, can be more easily adjusted by policy makers through pesticide taxes.

Effect of overall indicator accuracy (AUC). As one might expect, better predictors (i.e., those with high values of AUC) gave better values for $C R$ compared with poorer predictors, and indicators with modest accuracy are interesting only in a small range of Prev (i.e., in a small number of production situations). The performance of the indicator also affected the range of Prev values where it would be effective, and high-quality indicators can significantly improve the financial advantage of using $C R$ over a wide range of Prev (i.e., in a large number of production situations). An increase of the accuracy of the predictor beyond a value of $\mathrm{AUC}=0.9$ would further increase the prevalence range where it might be effective; however, this could be difficult (if not impossible) to achieve in practice.

The FAO suggests, as a rule of thumb, that adoption of an innovation is unlikely unless it offers at least a 2:1 financial advantage over existing practices, corresponding to a value of $C R$ of 0.5 . Even if this view may be a little pessimistic (23), our calculations indicate that such a $C R$ is never reached even with the highest quality indicator $(\mathrm{AUC}=0.9)$. This may be a possible explanation for the low rate of adoption of decision rule by farmers. Hopefully, future progress in plant disease epidemiology will lead to more accurate decision rules. If this is the case, then decision rule strategies will be more widely adopted because both their financial advantage and the range of production situations where they are of interest will increase. However, because of the inescapable trade-offs between the accuracy of decision rules and their userfriendly characteristics, it is important to keep in mind that even the most accurate decision rule will not be used if it requires too much effort on the part of the user (7). Noise limitations in terms of the biological system and the availability of data also will limit the quality of indicators. Accordingly, this result suggests that decision rules should be marketed to farmers by focussing on the cost savings and not by promoting large benefits over an existing practice.

The analyses done throughout this article are based on two premises. First, the potential usefulness of decision rules is compared against the better of the systematic strategies. This is because our main concern is not to investigate how decision rules can lead to reduced use of pesticides but rather how decision rules can lead to better targeted use of pesticides. Although the implementation of decision rules is often motivated by the reduction of 
the number of unnecessary treatments, there is also the opposite situation (referred as the management of moderately destructive pathogens in extensive crops) (31), where farmers tend not to spray at all and, consequently, where crops are not protected even in the case of major yield losses. Here, we consider that both situations are obviously undesirable. Second, it is assumed that farmers judge the performance of control strategies only on the basis of the relative average $C R$. This is true under the risk-neutral framework. Emphasis in this article has been placed on this situation because it provides a reference to interpret the level of profitability of control strategies (34). However, many decision makers are not strictly risk neutral (27). For example, in the case of destructive pathogens in intensive crops (greenhouse crops, for example), many farmers choose their control strategy by considering the absolute value of the crop rather than relative costs (31). Economists have used utility functions to take into account both the financial and psychological aspects of decision making; this general concept describes in what sense a decision maker will consider that a decision is optimal (35). The farmer's main concern often is not only to minimize the average cost of their control strategy (the profitability component of the utility function) but also to avoid extremely large variation (the risk component). In this case, their objective then is to maximize some function of the mean and the variance of the expected cost such as the incomevariance criterion (8). The financial interest of using a decision rule strategy will deviate more or less from our results depending on the priority given to the profitability component relative to the risk component. Application of subjective criteria is outside the scope of this study. However, in general, the more farmers tend to favor the risk component (in being either risk adverse or risk prone), the more they will favor the financial interest of systematic strategies. Finally, in this work, it is assumed that using the decision rule strategy induces no overhead costs. When this is not the case (for example, scouting for insects in the field is time consuming, decision rule recommendation are sometimes delivered only by subscription, and so on), the financial interest of using a decision rule will be lower than expected here.

The main interest of this work is to provide quantitative guidelines on the financial advantage that decision rules can offer in plant protection and, more generally, a better understanding of their potential usefulness. As noticed by Yuen and Hughes (39), knowing the potential performance of decision rules is crucial because it will enable targeting of areas (i.e., production situations) where there is a chance that it might be used. The methodology proposed here constitutes a general framework where one can a priori assess the potential interest of investing money and time to implement new decision rules or to improve existing ones. It mainly requires one to characterize the response of a pathosystem to the considered control method through some basic parameters (e.g., Cmin, Cmax, Ctrt, and Prev). This can be done simply from a set of yield measurements obtained on treated and untreated fields plots. Such data can be collected from pesticide efficacy trials commonly carried out by extension services and research institutes. Even if this study focuses particularly on the control of pathogens (pests) solely with the use of pesticides, both methodology and main conclusions can be easily extended to other control methods (e.g., biological control or resistant cultivars) where binary decisions are involved.

\section{ACKNOWLEDGMENTS}

We thank P. Debaeke for providing data on sunflower phoma, M. Taverne and D. Makowski for providing data on oilseed rape Sclerotinia spp., and to S. Lemarié for a critical reading of the manuscript.

\section{LITERATURE CITED}

1. Debaeke, P., and Chabanis, J. 1999. An integrated simulation model of yield loss by a fungal pathogen: Stem canker (Phomopsis helianthi) in sunflower. Pages 73-74 in: Proc. 1st Int. ESA Symp. Modelling Cropping Syst. Lleida, Spain.

2. Dent, D. 2000. Insect Pest Management. CABI, UK.

3. Dewdney, M. M., Biggs, A. R., and Turechek, W. W. 2004. A statistical comparison of MARYBLYT and Cougarblight with Receiver Operating Characteristic (ROC) curve analysis. Page 41 in: Proc. Int. Fire Blight Conf. Bologna, Italy.

4. De Wit, C. T., and Penning de Vries, W. W. T. 1982. L'analyse des systèmes de production primaires. Pages 275-283 in: La Productivité des Pâturages Sahéliens. W. W. T. Penning de Vries and M. A. Djiteye, eds. Pudoc, Wageningen, The Netherlands.

5. Fabre, F. 2004. Mise au point d'un modèle d'aide à la decision pour raisonner les traitements contre les vecteurs de la jaunisse nanisante de l'orge à l'automne sur céréales. Thesis, Agrocampus Rennes-Université de Rennes, France.

6. Fabre, F., Dedryver, C. A., Leterrier, J. L., and Plantegenest, M. 2003. Aphid abundance on cereals in autumn predicts yield losses caused by Barley yellow dwarf virus. Phytopathology 93:1217-1222.

7. Gordon, S. E., Gill, R. T., and Dingus, T. A. 1987. Designing for the user: The role of human factors in expert system development. AI Appl. Environ. Sci. 1:35-46.

8. Hazell, P. B. R. 1971. A linear alternative to quadratic and semivariance programming for farm planning under uncertainty. Am. J. Agric. Econ. 53:53-62.

9. Higley, L. G., and Wintersteen, W. K. 1992. A new approach to environmental risk assessment of pesticides as a basis for incorporating environmental costs into economic injury levels. Am. Entomol. 38:34-39.

10. Hughes, G. 1999. Sampling for decision making in crop loss assessment and pest management: introduction. Phytopathology 89:1080-1083.

11. Hughes, G., and Madden, L. V. 2003. Evaluating predictive models with application in regulatory policy of invasive weeds. Agric. Syst. 76:755774.

12. Hughes, G., McRoberts, N., and Burnett, F. J. 1999. Decision-making and diagnosis in disease management. Plant Pathol. 48:147-153.

13. Jeger, M. J. 2004. Analysis of disease progress as a basis for evaluating disease management practices. Annu. Rev. Phytopathol. 42:61-82.

14. Knight, A. L., and Norton, G. W. 1989. Economics of agricultural pesticide resistance in arthropods. Annu. Rev. Entomol. 34:293-313.

15. Knight, J. D. 1997. The role of decision support systems in integrated crop protection. Agric. Ecosyst. Environ. 64:157-163.

16. Knight J. D., and Mumford, J. D. 1994. Decision support systems in crop protection. Outlook Agric. 23:281-285.

17. Larsson, H. 2005. A crop loss model and economic thresholds for the grain aphid, Sitobion avenae (F.), in winter wheat in southern Sweden. Crop Prot. 24:397-405.

18. Lindblad, M. 2001. Development and evaluation of a logistic risk model: predicting frit fly infestation in oats. Ecol. Appl. 11:1563-1572.

19. Lloyd, C. J. 2000. Regression model for convex ROC curve. Biometrics 56:862-867.

20. Madden, L. V., and Ellis, M. A. 1988. How to develop plant disease forecaster. Pages 191-208 in: Experimental Techniques in Plant Disease Epidemiology. J. Kranz and J. Rotem, eds. Springer, New York.

21. Makowski, D., Taverne, M., Bolomier, J., and Ducarne, M. 2004. Comparison of risk indicators for Sclerotinia control in oilseed rape. Crop Prot. 24:527-531.

22. McCown, R. L. 2002. Changing systems for supporting farmers' decisions: problems, paradigms, and prospects. Agric. Syst. 74:179-220.

23. McRoberts, N., Hughes, G., and Savary, S. 2003. Integrated approaches to understanding and control of diseases and pests in field crops. Aust. Plant Pathol. 32:167-180.

24. Metz, C. E. 1978. Basic principles of ROC analysis. Semin. Nucl. Med. 8:283-298

25. Metz, C. E. 1986. ROC methodology in radiologic imaging. Invest. Radiol. 21:720-733

26. Meynard, J. M., Doré, T., and Lucas, P. 2003. Agronomic approach: cropping systems and plant diseases. C. R. Biol. 326:37-46.

27. Mumford, J. D., and Norton, G. A. 1984. Economics of decision making in pest management. Annu. Rev. Entomol. 29:157-174.

28. Murtaugh P. A. 1996. The statistical evaluation of ecological indicators. Ecol. Appl. 6:132-139.

29. Pedigo, L. P., and Higley, L. G. 1992. The economic injury level concept and environmental quality. A new perspective. Am. Entomol. 92:12-21.

30. Savary, S., Willocquet, L., Elazegui, F. A., Castilla, N. P., and Teng, P. S. 2000. Rice pest constraints in tropical Asia: quantification of yield losses due to rice pests in a range of production situations. Plant Dis. 84:357369.

31. Shtienberg, D. 2000. Modelling: the basis for rational disease management. Crop Prot. 19:747-752.

32. Swets, J. A. 1988. Measuring the accuracy of diagnostic systems. Science 240:1285-1293. 
33. Swets, J. A., Dawes, R. M., and Monahan, J. 2000. Better decisions through science. Scientific Am. 283:70-75.

34. Rossing, W. A. H., Daamen, R. A., and Hendrix, E. M. T. 1994. Framework to support decisions on chemical pest control under uncertainty, applied to aphids and brown rust in winter wheat. Crop Prot. 13:25-34.

35. Teng, P. S., and Yuen, J. E. 1991. Epidemics models: Lessons from plant pathology. Pages 272-296 in: Risk Assessment in Genetic Engineering. M. A. Levin and H. S. Strauss, eds. McGraw Hill, New York.

36. Turechek, W. W., and Wilcox, W. F. 2005. Evaluating predictors of apple scab with receiver operating characteristic curve analysis. Phytopathology
95:679-691.

37. Way, M. J., and van Emden, H. F. 2000. Integrated pest management in practice-pathways towards successful application. Crop Prot. 19:81-103.

38. Wearing, C. H. 1988. Evaluating the IPM implementation process. Annu. Rev. Entomol. 33:17-38.

39. Yuen, J. E., and Hughes, G. 2002. Bayesian analysis of plant disease prediction. Plant Pathol. 51:407-412.

40. Yuen, J. E., Twengström, E., and Sigvald, R. 1996. Calibration and verification of risk algorithms using logistic regression. Eur. J. Plant. Pathol. 102:847-854. 